\title{
Self-Evaluated Evidence-Based Medicine Skills Improve among Last Year Medical Student, within Nine Weeks, When Introducing a Blended EBM Teaching Approach
}

\author{
Daniel Novak*, Már Tulinius \\ Department of Paediatrics, Queen Silvia Children’s Hospital, Sahlgrenska Academy, University of Gothenburg, Gothenburg, Sweden \\ *Corresponding author: daniel.novak@gu.se
}

Received April 10, 2015; Revised April 17, 2015; Accepted April 22, 2015

\begin{abstract}
The practice of evidence-based medicine (EBM) is important for medical students to master to optimize their future clinical decision-making and improve the quality of health care. The aim of this study was to explore Swedish final-year medical students' self-evaluated EBM skills and to evaluate the effect of actively teaching EBM during a nine-week pediatric course. A control group $(n=39)$ and an intervention group $(n=44)$ consisted of last year medical students enrolled in a nine week pediatric course. Both groups only differed by the year of starting the studies: year 2012 vs year 2013. Students anonymously self-evaluated their EBM skills at the beginning and at the end of the course. The intervention consisted of blended EBM teaching by medical teachers in both class room and clinical settings. The control group did not receive an intervention. The participation rate in the intervention group was 91\% (40/44) and in the control group 75\% (29/39). Students' overall baseline EBM skills score was 5.1 (on a scale of 10) in the intervention group and 5.5 in the control group. After the intervention, searching for EBM increased by 1.45 (95\% CI: 0.78-2.11), critically appraising EBM increased by 1.51 (95\% CI: 0.88-2.15), and applying EBM in patient care increased by 2.08 (95\% CI: 1.46-2.70). The findings show that many final-year Swedish medical students rate their own EBM skills as low. However, it is possible with a small investment of time from the medical teacher during a nine-week pediatric course to significantly improve students' self-evaluated EBM skills.
\end{abstract}

Keywords: Evidence-based medicine skills, medical education, intervention, Sweden

Cite This Article: Daniel Novak, and Már Tulinius, "Self-Evaluated Evidence-Based Medicine Skills Improve among Last Year Medical Student, within Nine Weeks, When Introducing a Blended EBM Teaching Approach." American Journal of Educational Research, vol. 3, no. 5 (2015): 594-598. doi: 10.12691/education-3-5-10.

\section{Introduction}

The practice of evidence-based medicine (EBM), which implies finding and appraising clinical studies critically and considering their application to clinical practice, aims to stimulate lifelong learning and educate the students to become physicians with an ability to use EBM independently in their future practice. It is shown to be vital that physicians incorporate EBM practice in their professional life to stay up to date and deliver optimal health care [1].

Increasingly, EBM has been included in the curriculum for medical courses worldwide, at both graduate and undergraduate levels and in both clinical and non-clinical settings [2,3,4,5]. However, studies have identified several barriers to the implementation of EBM at the undergraduate level. Lack of time and access to appropriate technology are barriers to how students integrate EBM practice into their daily study $[2,4,6]$. Lack of time and integrated curriculums are barriers for teaching EBM in clinical settings [3,7]. In certain countries, it has been shown that the lack of understanding of English, and the time required for reading English language articles, are barriers for both students and teachers in using EBM in daily practice [5].

Adopting an EBM approach requires that the users understand and apply five basic steps: 1) Constructing a clinical question 2) Acquire the evidence via a systematic and efficient search of the literature 3) Critically appraising the literature 4) Applying the evidence to a clinical scenario 5) Assessing the EBM process [8]. Each step requires a different knowledge. A systemic review evaluating EBM teaching methods showed that the classical didactic teaching method improved students' knowledge but not their skills, attitudes or behavior in EBM [9]. A blended learning approach has been advocated as an EBM teaching method that promotes greater student appreciation of the EBM principles within the clinical setting [10].

Our study evaluates last year medical students EBM experiences and skills and attempts to overcome the barriers of implementing EBM teaching at the undergraduate level. The effect of introducing a blended EBM education during a nine week pediatric course upon students' self-assessed EBM skills is evaluated by a controlled study. 


\section{Method}

\subsection{Design/setting}

The study was conducted during the year 2012 and the year 2013 at the University of Gothenburg Medical School in Sweden, Queen Silivas Childrens' Hospital. One group received active EBM teaching and the other group received no EBM teaching during pediatric course.

\subsection{Participants}

Participants were recruited among last year medical student admitted to a nine week pediatric course. Both groups only differed by the year of starting the studies: year 2012 for the interventions group and the year 2013 for the control group (Table 1).

Table 1. Self evaluated EBM skills.

\begin{tabular}{|c|c|c|c|c|}
\hline Characteristic & $\begin{array}{c}\text { All } \\
(n=69)\end{array}$ & $\begin{array}{l}\text { Intervention group } \\
(\mathrm{n}=40)\end{array}$ & $\begin{array}{c}\text { Control group } \\
(\mathrm{n}=29)\end{array}$ & P-value \\
\hline Female & $43(62 \%)$ & $22(55 \%)$ & $21(72 \%)$ & NS \\
\hline Age in years (average) & 27.5 & 28 & 27 & NS \\
\hline Number of students who are parents & $9(13 \%)$ & $6(15 \%)$ & $3(7,5 \%)$ & NS \\
\hline Previously worked as a physician & $61(88 \%)$ & $37(93 \%)$ & $24(83 \%)$ & 0.04 \\
\hline Previously used EBM in clinical practice & $29(42 \%)$ & $16(40 \%)$ & $13(35 \%)$ & NS \\
\hline Average number of EBM articles used while working as a physician & 2.1 & 1.6 & 2.6 & NS \\
\hline Average number of EBM articles used in medical school & 5.5 & 4.6 & 6.5 & NS \\
\hline Total amount of knowledge gained in the pediatric course from the Internet \% & 38.5 & 38 & 39 & NS \\
\hline
\end{tabular}

\subsection{Data Collection}

Prior to starting the course both groups had to answer a baseline questionnaire, self-assessing their own EBM skills. The design of the questionnaire was based on the five basic steps of adopting an EBM approach [8] and a total of five questions were taken from the Taipei Evidence-Based Practice Questionnaire (TEBPQ). TEBPQ is an instrument that has been shown to have good validity and reliability for evaluating the effectiveness of EBM education [11]. A 10-point Likert scale was used where 1 corresponded to 'very insecure' and 10 corresponded to 'excellent'. The questionnaire also contained questions regarding their demographic background and prior experience of EBM. A total of 10 questions were included in the questionnaire to make the questionnaire small and increase the participation rate.

Finally, during the last day of the course, the students in the intervention group and the control group anonymously self-evaluated their own EBM skills by completing the same coded questionnaire used at the beginning of the course. An exclusion criterion in both the intervention group and the control group was being an exchange student, who had not previously studied or worked in Sweden.

\subsection{Intervention}

In the intervention class a blended EBM teaching approach was performed in both students' rooms and in clinical settings. During the first day of the course the students received a 40 minute lecture from a medical teacher actively working in the pediatric ward (one of the authors). The purpose of the lecture was to teach the students how a clinical pediatrician searches for EBM literature. The teacher described how to search effectively in the following databases: PubMed, UpToDate, BestPractice, Cochrane, and SBU (independent national authority assessing health care interventions covering medical, economic, ethical and social aspects) [12]. The students were taught how to access these databases from both the hospital computers and their home computers. A medical librarian was present during the whole lecture and answered questions when necessary. Throughout the course the student had access to a student computer room with a printer in the hospital. During the course the students were requested to complete two EBM tasks. Both tasks required the students to construct a clinical question based on a clinical scenario, identify relevant literature that answers the question, critically appraise the literature, and suggest an implementation of their findings in the clinical scenario.

The first task was to evaluate a one pediatric patient in the outpatient clinic during the course. The patient came by referral, knowing that s/he would meet a medical student under supervision. The supervisor was a medical teacher (one of the authors). The medical student received the patient referral form one week in advance, which allowed them to study the text book literature of the possible disease. The students were also asked to search the latest knowledge on the disease using the databases previously mentioned. The students were requested to solve the clinical question by using their EBM skills and bring one printed copy of their article on the day of their outpatient clinic. After the clinical consultation the student would present his/her article, appraise it critically and consider its application to clinical practice. Finally, the student would get both clinical feedback and feedback on their EBM skills from the medical teacher.

The second task required each student to identify an interesting patient case from their clinical rounds and present it to a group of fellow students applying their EBM skills. This was done in a student-room setting with two medical teachers present (both authors) who merely acted as facilitators. The purpose of the presentation was not only to teach their fellow students but also stimulate a discussion about clinical treatment. The student had to send the EBM article a day before the presentation to both medical teachers. The students presented the article, appraised it critically, and considered its application to clinical practice for five minutes. The students then received feedback on their presentation and their EBM skills. The control group did not receive any EBM education.

\subsection{Ethics}

The regional ethics committee (University of Gothenburg) did not believe that an ethical approval was necessary for this study. 


\subsection{Statistical Analysis}

Differences in the distribution of background characteristics were examined using Chi-square tests for categorical variables and Student's t-tests for continuous variables. All p-values were two-tailed and values $<0.05$ were considered significant. Differences in the pre and post assessment rating were examined using the paired sample t-test, and a 95\% confidence intervals (CI) was used to express the significance level. Statistical analyses were performed using SPSS, Windows version 18.0 (SPSS Inc., Chicago, IL, USA).

\section{Results}

Of the eligible students, 91\% (40/44) in the intervention group and $75 \%$ (29/39) in the control group answered both questionnaires. The characteristics of the intervention and the control group are presented in Table 1 . There was no gender difference within the groups. A small number of EBM articles had previously been used during both medical school and while working as a physician. Overall internet use was similar between the groups with an average of $38.5 \%$ (range 5-90\%) of the total course knowledge received from the internet. More students in the intervention group than in the control group had worked as a physician during the previous summer, which is allowed in Sweden after the fourth year of medical school.

The overall baseline average score of EBM skills was 5.1 in the intervention group. In the intervention group all self-evaluated EBM skills increased significantly after the course (Table 2).

Table 2. Self evaluated EBM skills in the intervention group before and after the pediatric course $n=40$.

\begin{tabular}{|c|c|c|c|c|}
\hline Variable & Pre-course assessment & Post-course assessment & Change in assessment score (95\% CI) & P-value \\
\hline The importance of EBM to your future career & 8.12 & 8.82 & $0.70(0.23-1.15)$ & 0.04 \\
\hline Searching for EBM & 5.83 & 7.28 & $1.45(0.78-2.11)$ & 0.001 \\
\hline Critically appraising EBM & 5.03 & 6.54 & $1.51(0.88-2.15)$ & 0.001 \\
\hline Applying EBM in patient care & 4.36 & 6.44 & $2.08(1.46-2.70)$ & 0.001 \\
\hline
\end{tabular}

Note: The self reported scale ranges from 1 to 10 where 10 correspond to 'excellent or very important'.

In the control group, which consisted of medical students participating in the same course exactly one year later but who did not receive any intervention, there was no significant change in self-evaluated EBM skills (Table

3). The overall average score of EBM skills was 5.5 in the control group, which did not differ significantly from the intervention group.

Table 3. Self evaluated EBM skills in the control group before and after the pediatric course $\mathbf{n}=\mathbf{2 9}$.

\begin{tabular}{|c|c|c|c|c|}
\hline Variable & Pre-course assessment & Post-course assessment & Change in assessment score (95\% CI) & P-value \\
\hline The importance of EBM to your future career & 8.83 & 8.48 & $-0.35(-0.17-0.86)$ & $\mathrm{ns}$ \\
\hline Searching for EBM & 6.69 & 7.68 & $0.99(-0.19-1.78)$ & $\mathrm{ns}$ \\
\hline Critically appraising EBM & 5.34 & 6.10 & $0.76(-0.42-1.40)$ & $\mathrm{ns}$ \\
\hline Applying EBM in patient care & 4.55 & 5.25 & $0.71(-0.54-2.70)$ & $\mathrm{ns}$ \\
\hline
\end{tabular}

The time taken for the complete intervention was one hour of preparation with the medical librarian to learn about the latest updates and searching techniques for the EBM databases and 40 minutes lecture for the students. In the outpatient clinic scenario the discussion about the student's EBM article usually took about five minutes. The medical teacher read the abstract of the article while observing the student-patient consultation. In the studentroom scenario the discussion about the EBM article usually took 10 minutes instead of the requested 5 minutes. These student articles were read more thoroughly the day before by both medical teachers.

\section{Discussion}

With the current rapid influx of new research, medical literature books quickly become outdated. It is therefore vital that medical students learn how to efficiently find and apply the latest knowledge to their patients. EBM equips the doctor with knowledge of how to integrate evidence from research into clinical decision-making and thereby improve the quality of health care.

\subsection{Improvement of the Students' EBM Skills}

The study showed that many final-year medical students rated their pre-course EBM skills as average. At the end of a medical school, (which has, according to the curriculum, incorporated EBM into the education from the beginning) the overall average score of self-evaluated EBM skills was 5.1 (on a scale of 10) in the intervention group and 5.5 in the control group. After graduation regular EBM teaching in clinical practice is rare if journal clubs are excluded $[7,13]$, thus it is very important that students feel confident about their EBM skills and incorporate them into their daily routine before they leave the university.

In this study we demonstrated a positive impact of actively teaching EBM during a nine-week pediatric course. After the using a blended EBM teaching approach in the intervention group their overall self-evaluated EBM skills increased significantly. Even though many students rated their own skills as low and the course was at the very end of their medical education, a small amount of extra teacher effort improved their self-evaluated EBM skills. Searching and critically appraising EBM was a skill that the students were most confident in, suggesting that they had learned and practiced this skill from previous workshops during their education. Throughout their medical education each student had been involved in a research project and written a research report, which had been approved by a supervisor. During this research period they had actively practiced searching and critically appraising scientific literature.

However, applying EBM in patient care was the variable that improved the most after the intervention 2.08 (95\%CI 1.46-2.70). Applying EBM is the most important 
step, which involves turning a clinical problem into an answerable question and, with the help of EBM literature, answering the question and applying it to the patient $[14,15,16]$. Applying EBM in patient care is also a skill that can only be practiced during the clinical rotation in medical school. In Swedish medical schools clinical rotation begins from the middle of the third year where the main focus is the student-patient interaction. Therefore in a busy ward it may be common that EBM practice with medical students is forgotten.

Many students rated the importance of learning EBM for their future practice as very high (8.1 in the intervention group and 8.8 in the control group) before the course. After the intervention this belief increased by 0.70 (95\% CI 0.23-1.15) to 8.81 (p-value 0.04). It is promising that students value EBM, and it is also promising that active teaching and promotion of EBM by the clinical teacher increases the students' interests and awareness of EBM.

A lack of understanding of English and the time required for reading English language articles have been cited as barriers for students using EBM [5]. However, we did not encounter any language difficulties throughout the course and all students correctly understood the EBM articles. English proficiency is very high among Swedish medical students and many medical course books are in English [17].

\subsection{Previous EBM Experience}

The study showed that many final-year medical students rated their pre-course EBM skills as medium both in the intervention and in the control class. Previous studies have also shown similar results among graduating medical students (Lypson 2004, Caspi 2004). It is surprising that an average of only 5.5 EBM literature articles were previously used during the student's medical school education. EBM is implemented from the first year in medical school and numerous group assignments which include EBM literature are given to students throughout their studies. The reason for this low number could be that the students did not remember these exercises, or they did not consider them as EBM because they may have been used in the pre-clinical period of medical school. Whatever the reason, if EBM is implemented throughout medical education it is important to emphasize this to students and accustom them to the fact that they are using their EBM skills. It is gratifying that an average of 2.1 EBM articles were used during their previous summer's work as doctors-under-supervision. This could be a sign of a maturated learning process. Adult learning theory suggests that the determinants of learning among postgraduates are driven by self-motivation and relevance to clinical practice, whereas undergraduate learning is driven by external factors such as curriculum and examinations [18]. The use of EBM during previous medical work could also be a sign of a clinic that has successfully incorporated EBM into daily practice. Teaching EBM in everyday practice to junior doctors is uncommon and has been shown to be a challenge $[7,13,19]$, but it may be that if EBM is introduced early in medical school education and practiced regularly these skills and behaviors may be transferable to clinical practice.

\subsection{The Time Consumption of the Intervention}

The introduction lecture to searching for EBM literature took 40 minutes. The preparation before the lecture with the librarian took two hours. In the future a 30-minute preparation time should be sufficient to update the teacher on the latest EBM databases with the librarian. An alternative is to have the whole lecture undertaken by the librarian, but the purpose, for the students, is to see how a medical doctor searches for EBM literature.

During the outpatient clinic the student brought a printed copy of his/her EBM article with them. The medical teacher briefly read through the abstract of the article during the student-patient consultation. The EBM discussion with the student after the consultation took about 5-10 minutes where the purpose was to stimulate the student to think critically about how the results could be applicable to the patient's care.

In the student-room scenario the teacher needed to prepare beforehand by reading the article mailed to them before the presentation. An estimated 10 minutes of reading time for the article and 5 minutes to stimulate the EBM discussion was required from the medical teacher. The intervention therefore required one lecture and a maximum amount of time of 25 minutes per student. Practicing EBM during the medical course not only improves the medical student's EBM skills but also those of the teacher (authors note).

Therefore, effectively integrating EBM into the curriculum, supplying appropriate technological access for students and requires a slight increasing in teaching effort (25 minutes per student) but leads to a significant increase in the students' self-assessed EBM skills.

\subsection{Limitations of the Study}

The study has several limitations. The study sample is relatively small, which may limit its statistical power. However, the confidence intervals exclude zero indicates that the effect of the intervention is reasonably large.

Unfortunately, it is unknown how long the effect of the intervention will last. It would be of interest to re-evaluate the students after one year of practicing medicine and see if their self-evaluated EBM skills persist. Searching for EBM and applying it to patients is a skill that needs to be practiced regularly [15]. During the progression of the course, the course directors received feedback from the clinical teachers on the ward that many students were absent from the ward claiming that they had to prepare for the presentation and find EBM articles. Whether this was a reason to skip the ward rotation, or whether EBM was actually time consuming and difficult for some, was not investigated further.

A further limitation of the study is the evaluation process. We performed a comparison of traditional pre/post self-assessments in both the intervention and the control group. A traditional pre/post self-assessment has been used in numerous EBM studies previously (Nicholson 2007, Lai 2010, Liabsetrakul 2009) However, it is suggested that retrospective pre/post self-assessment compared to traditional pre/post self-evaluation may provide a more sensitive and more valid measure of the learning outcomes in an intervention study [20]. When comparing these two evaluation methods the means of the 
pre-score using the traditional pre/post self-assessment method were consistently and significantly higher than the means using the retrospective self-assessment method [20]. There is, therefore, a risk of underestimating the effect of the intervention by using traditional pre/post selfassessment instead of retrospective pre/post selfassessment. [21,22].

Some researchers have argued that high levels of selfreported competence and preparedness are correlated with high performance (Shubert 1999) other have doubted whether self-assessment is a valid predictor of true competence (Gordon 1991, Ward 2002, Caspi 2006). Even though the students in the intervention class significantly improved their self-assessment score, relying only on medical students' self-perceived confidence is not enough. Therefore, further research that objectively evaluates students' EBM skills is needed.

\section{Conclusions}

Many final-year Swedish medical students rate their own EBM skills as low but they believe that EBM is an important tool in their future practice. However, it is possible by effectively integrating a blended EBM teaching approach into the curriculum, to significantly improve last year medical students' self-evaluated EBM skills within nine weeks

\section{Acknowledgement}

The authors wish to thank medical librarian Eva-Lotta Daxberg for preparation and assistance during the introduction lecture.

\section{Declarations of Interest}

The authors have no declarations of interest to report.

\section{References}

[1] Raza A, Coomarasamy A, Khan KS. Best evidence continuous medical education. Arch Gynecol Obstet. 2009; 280 (4): 683-7.

[2] Bradley P, Oterholt C, Nordheim L, Bjorndal A. Medical students' and tutors' experiences of directed and self-directed learning programs in evidence-based medicine: a qualitative evaluation accompanying a randomized controlled trial. Eval Rev. 2005; 29 (2): 149-77.
[3] Finkel ML, Brown HA, Gerber LM, Supino PG. Teaching evidence-based medicine to medical students. Med Teach. 2003; 25 (2): 202-4.

[4] Ilic D, Forbes K. Undergraduate medical student perceptions and use of Evidence Based Medicine: a qualitative study. BMC Med Educ. 2010; 10: 58.

[5] Oude Rengerink K, Thangaratinam S, Barnfield G, Suter K, Horvath AR, Walczak J, et al. How can we teach EBM in clinical practice? An analysis of barriers to implementation of on-the-job EBM teaching and learning. Med Teach. 2011; 33 (3): e125-30.

[6] Khanjani NT, R. Maghsoudi, A. The Obstacles of Teaching Evidence Based Medicine in Iran, from the View Point of Clinical Academics and Medical Students; a Qualitative Study. American Journal of Educational Research. 2013; 1 (4): 6.

[7] Hatala R, Keitz SA, Wilson MC, Guyatt G. Beyond journal clubs. Moving toward an integrated evidence-based medicine curriculum. J Gen Intern Med. 2006; 21 (5): 538-41.

[8] Straus S GP, Richardson W, Haynes R. Evidence-based medicine. How to practice and teach it. Toronto: Churchill Livingstone Elsevier; 2011.

[9] Coomarasamy A KK. What is the evidence that postgraduate teaching in evidence based medicine changes anything? A systematic review. BMJ. 2004; 329 (7473): 1017.

[10] Ilic D, Hart W, Fiddes P, Misso M, Villanueva E. Adopting a blended learning approach to teaching evidence based medicine: a mixed methods study. BMC Med Educ. 2013; 13: 169.

[11] Chen KT, PC. Chen, TH. Kuo, KN. Liu, HE. Chen, C. Develop a Self-Evaluation Questionnaire for Evidence-Based Practice Education American Journal of Educational Research. 2014; 2 (9): 5.

[12] Swedish Council in Health Technolgy Assessment [Internet]. http://www.sbu.se/en/. 2013. Available from: http://www.sbu.se/en/.

[13] Dawes M, Summerskill W, Glasziou P, Cartabellotta A, Martin J, Hopayian K, et al. Sicily statement on evidence-based practice. BMC Med Educ. 2005;5(1):1.

[14] EBMWG. Evidence-based medicine. A new approach to teaching the practice of medicine. JAMA. 1992; 268 (17): 2420-5.

[15] Sackett DR, WS. Rosenberg, W. Haynes, RB. Evidence-based medicine: how to practice and teach EBM. Haynes R, editor. London: Churchill Livingstone,; 2000.

[16] Sweeney K. How can evidence-based medicine help patients in general practice? Fam Pract. 1996; 13 (6): 489-90.

[17] Gunnarsson B. Swedish, English, French or German- tha language sitaution at Swedish Universities. In: Ammon U, editor. The dominance of English as a language of science. Berlin: Mouton de Gruyter; 2001.

[18] Knowles MS DC, Basford P. Teaching and assessing in clinical practice. London: University of Greenwich; 1998. p. 23-38.

[19] van Dijk N, Hooft L, Wieringa-de Waard M. What are the barriers to residents' practicing evidence-based medicine? A systematic review. Acad Med. 2010; 85 (7): 1163-70.

[20] Skeff KS, G. Bergen, M. Evaluation of a medical faculty development program. A comparison of traditional pre/post and retrospective pre/post self-assessment ratings. Eval Health Prof. 1992; 15: 350-66.

[21] Bray JH, G. Methodological considerations in the evaluation of a teacher training program. J Educ Psych. 1980; 72: 62-70.

[22] Levinson WG, G. Skeff,K. Rerospective versus actual pre-course self-assessments. Eval Health Prof. 1990; 13: 445-52. 